\title{
Follow-up of patients with suspected multiple sclerosis: a clinical and electrophysiological study
}

\author{
J BØTTCHER, W TROJABORG \\ From the Departments of Neurology and Clinical Neurophysiology, University Hospital, Rigshospitalet,
Copenhagen, Denmark
}

SUMMARY We re-examined 21 patients with suspected multiple sclerosis, seven classified as probable and 14 as possible cases. At the first investigation all patients but two had abnormal visual evoked potentials and somatosensory evoked potentials, or both. All but three had an increased intracerebral production of immunoglobulin $G$ expressed by the cerebrospinal fluid IgG index. At follow-up two to four years later, 13 of 16 patients $(81 \%)$ in whom both evoked potentials and IgG index were abnormal initially had entered into a higher multiple sclerosis diagnostic class. In the five patients in whom either evoked potentials or IgG index were normal the original diagnosis was unchanged.

In a previous report ${ }^{1}$ we suggested a reclassification of patients with suspected multiple sclerosis when electrophysiological and CSF findings were taken into consideration. If abnormality of visual and somatosensory evoked potentials added more to the diagnosis than history and clinical signs and if the CSF immunoglobulin G (IgG) was increased, the patient was grouped in a higher class. To test the validity of this reclassification, we have undertaken a follow-up of the patients classified on clinical grounds as possible or probable cases of multiple sclerosis.

\section{Material and methods}

The patient group comprised 21 individuals ranging in age between 23 and 68 years (13 female, 8 male), who were considered to have probable ( 7 patients) and possible (14 patients) multiple sclerosis at the first examination according to previously published diagnostic criteria. ${ }^{2}$ In short, patients were classified as probable cases when they had had a single episode suggestive of multiple sclerosis and clinical evidence of lesions at two or more separate sites in the CNS (five patients), or a progressive history of paraplegia and clinical evidence of lesions at two or more separate sites in the CNS (two patients). Patients were classified as possible cases if they had had a progressive history of paraplegia and evidence of one lesion only (six patients),

Address for reprint requests: W Trojaborg, MD, Laboratory of Clinical Neurophysiology, University Hospital, Rigshospitalet, DK 2100, Blegdamsvej 9, Copenhagen, Denmark.

Received 10 January 1982 and in revised form 7 April 1982. Accepted 24 April 1982 or a single episode suggestive of multiple sclerosis with evidence of one lesion only (six patients), or without evidence of any lesion (two patients).

A re-examination took place $19-49$ months after the first investigation. In 18 cases the observation time was nore than two years. Cerebrospinal fluid (CSF) was obtained in all patients at the first examination. Determinations of albumin and IgG in CSF and blood serum were carried out as described elsewhere. ${ }^{1}$ The ratio of CSF serum IgG and CSF serum albumin, the IgG index, was used as a measure of the intracerebral production of IgG. At the first and final examination cortical potentials following pattern reversal stimulation and median and tibial nerve stimulation were recorded. In each patient stimulation was presented to each eye in turn and potentials were recorded over both occipital lobes. Similarly, stimuli were presented to the median and tibial nerves on each side in turn. The details of the techniques have been described elsewhere. ${ }^{134}$

\section{Results}

Figure 1 shows the CSF findings in the 21 patients. Three of the probable multiple sclerosis cases had a normal IgG index, one a slightly and three a moderately increased IgG index. All of the possible cases had a moderately or greatly increased IgG index.

The visual evoked potentials (VEPs) (table 1) were abnormal in the seven patients classified as probable multiple sclerosis cases, and in nine of the possible cases. The responses were delayed bilaterally in 12 and unilaterally in four patients. Three of the probable and one of the possible cases had had a history of optic neuritis. The VEP thus revealed subclinical optic nerve involvement in all but one of 


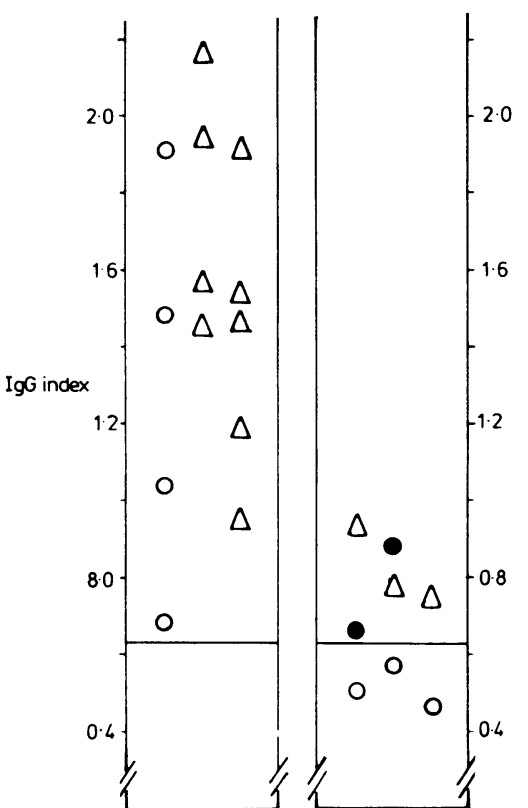

Fig 1 The individual values of the immunoglobulin $G$ index in patients with (left column) and without (right column) clinical deterioration. The open circles and triangles represent patients with probable and possible multiple sclerosis respectively who also had abnormal VEPs and SEPs or both. The closed circles indicate patients with normal EPs. The horizontal line denotes the upper normal limit of the IgG index.

the probable cases and in eight of the possible multiple sclerosis cases. The cortical response following median nerve stimulation was abnormal bilaterally in three of the probable and in eight of the possible cases. Unilateral abnormal somatosensory evoked potentials (SEPs) were found in one each of prob- able and possible cases; in eight patients the SEP was normal. The SEP following median nerve stimulation revealed subclinical involvement in eight possible multiple sclerosis cases; in four bilaterally and in four unilaterally. The SEP following tibial nerve stimulation was abnormal in all the probable cases, bilaterally so in six and unilaterally in one. Among the possible multiple sclerosis cases nine were abnormal on each side, one unilateral and four were normal. The SEP following tibial nerve stimulation revealed subclinical involvement in two probable and two possible cases (table 1).

We recorded six evoked potentials (EPs) in every patient and the number of abnormal EPs were related to the $\mathrm{IgG}$ index (table 2). Of 15 patients who had more than four abnormal EPs 12 had an increased $\mathrm{IgG}$ index. All patients with 2-3 or only one abnormal EP had similar CSF changes. The two patients with normal EPs had both a mildly increased IgG index.

Table 3 shows that four of the seven patients classified as probable multiple sclerosis cases at the first clinical examination all had developed new symptoms and signs of the disease during the follow-up period justifying a classification as definite multiple sclerosis cases. These four patients all gave a history of three or more episodes since the first investigation. All had clinical evidence of new separate lesions localised to the spinal cord, to the optic nerve in two patients and to the brain stem in one. All four had abnormal EPs and an increased IgG index, in three above $1 \cdot 01$. There was no clinical evidence of progression in the remaining three probable cases all of whom had a normal IgG index but abnormal EPs (fig 1).

Of the 14 possible multiple sclerosis cases 12 originally had abnormal EPs and an increased

Table 1 Incidence of abnormal evoked potentials in suspected multiple sclerosis cases at the first investigation

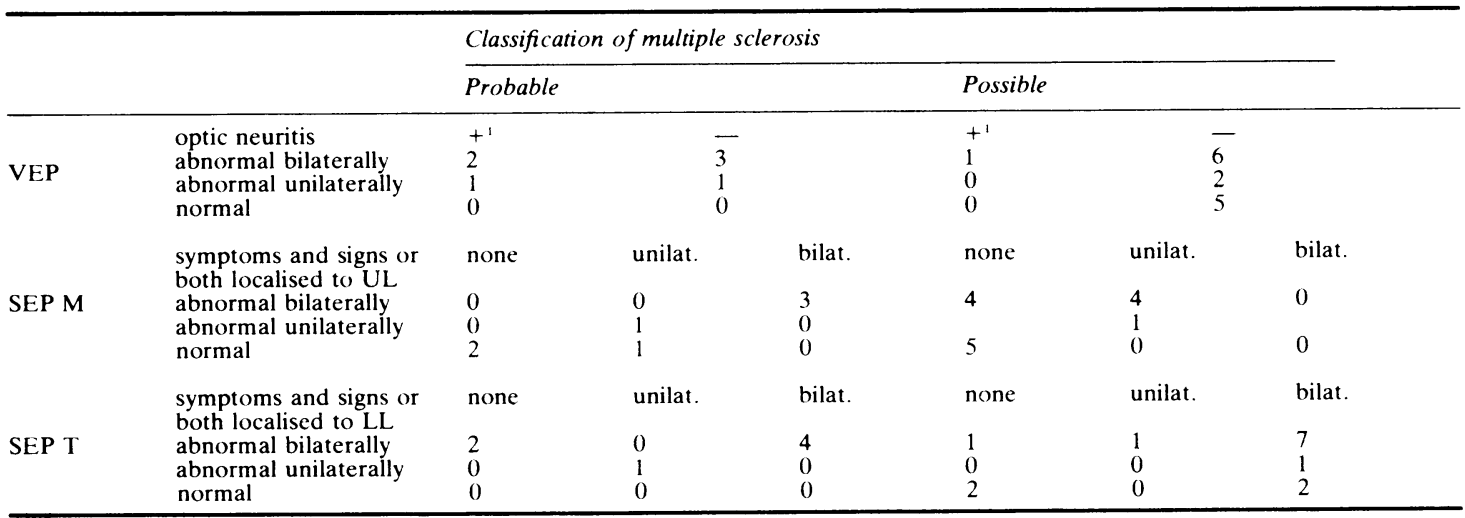

$1+=$ present (unilaterally in all), $-=$ absent

$\mathrm{M}=$ median nerve, $\mathrm{T}=$ tibial nerve, $\mathrm{UL}=$ upper limbs, $\mathrm{LL}=$ lower limbs 
Table 2 Comparison of EPs and CSF findings at the first examination of 21 suspected multiple sclerosis patients

\begin{tabular}{llll}
\hline No of abnormal EPS & \multicolumn{4}{l}{$C S F-\lg$ index } \\
\cline { 2 - 4 } & $\leq 0.63$ & $0.64-1.00$ & $\geq 1.01$ \\
\hline 0 & 0 & $2^{*}$ & 0 \\
1 & 0 & 0 & 1 \\
$2-3$ & 0 & 1 & 2 \\
$4-6$ & $3 \dagger$ & 5 & 7 \\
\hline
\end{tabular}

*Both classified as possible cases, fall classified as probable cases.

intracerebral IgG synthesis. Five of the 12 patients had new symptoms and signs and were classified as definite cases at the re-examination; all five had an IgG index above $1 \cdot 45$. Four of the five patients had clinical evidence of one lesion at the first examination after which they developed signs and symptoms of spinal cord and brain stem involvement and one, in addition, an optic neuritis. Four patients had progressed further and were considered probable cases, three of whom had an IgG index above 1.18. At the first examination they all had clinical evidence of one lesion only, but at the follow-up three patients had developed evidence of additional lesions localised to the spinal cord and brain stem, and one patient had a new lesion localised to the spinal cord. The clinical condition was unchanged in three with a slightly elevated IgG index. The remaining two possible multiple sclerosis cases who had normal EPs but slightly increased IgG index showed no progression clinically (fig 1 ). Of 10 patients with an IgG index below 1.0 two progressed clinically and eight did not, whereas all 11 patients with an IgG index above 1.0 progressed clinically $(p<$ $0.01)$. Thus, 13 of the 16 suspected multiple sclerosis cases $(81 \%)$ who had electrophysiological evidence of CNS lesions and an increased intracerebral IgG synthesis (moderately in 11 and slightly in 2) showed clinical evidence of progression, whereas three $(19 \%)$ did not. In these three cases there was a mild increase in IgG synthesis. The five suspected cases in whom only one of the examined parameters was abnormal (either EP or

Table 3 Follow-up of 21 patients with suspected multiple sclerosis

\begin{tabular}{lclll}
\hline $\begin{array}{l}\text { Classification at } \\
\text { first examination }\end{array}$ & No & \multicolumn{3}{l}{ Reclassification at follow-up } \\
\cline { 2 - 5 } & & Definite & Probable & Unchanged \\
\hline Probable & 4 & 4 & 0 & 0 \\
Possible & 12 & 5 & 4 & 3 \\
Total & $16^{*}$ & 9 & 4 & 3 \\
Probable & 3 & 0 & 0 & 3 \\
Possible & 2 & 0 & 0 & 2 \\
Total & $5 \dagger$ & 0 & 0 & 5 \\
\hline
\end{tabular}

*Patients with increased IgG and abnormal EPs at the first investigation, †patients with increased IgG and normal EPs or vice versa.
IgG index) showed no change in the clinical condition. The mean follow-up time for these five patients was 31 months (range 25-40).

Table 4 shows the average latency of EPs at the first and final investigation in the 13 patients in whom the disease progressed compared to the eight in whom the clinical condition was unchanged. On average the VEP latency increased from 116 to 127 $\mathrm{ms}$ in patients with clinical deterioration, whereas it was $108 \mathrm{~ms}$ and $109 \mathrm{~ms}$ at the first and final examination respectively in the patients without clinical progression. Regarding the average mean latency of SEPs following median nerve stimulation, there was no difference between the two examinations in either of the two groups. The mean latency of the SEP to tibial nerve stimulation increased from 46 to $58 \mathrm{~ms}$ in the group with clinical progression whereas no difference was found in the clinical unchanged group. An increase in latency of either the VEP or SEP or both occurred in 10 of the patients with clinical exacerbations and in only one of the clinically unchanged patients. Table 5 shows the average mean latency of EPs in patients with and without a change in latency from the first to the final investigation related to the clinical condition at the final examination. When the difference in latency between the two determinations exceeded twice the SD or more it was considered a significant change. There was an increase in the average latencies of the VEP and SEPs in 10 of the 13 patients with clinical progression, whereas the mean latency of EPs was unchanged in the eight patients without progression. The latency of P100 of the VEP increased from 118 to $141 \mathrm{~ms}(p<0.01)$ and the latency to onset of the SEPs following median and tibial nerve stimulation increased from 23 to $34 \mathrm{~ms}$ and from 49 to $73 \mathrm{~ms}$ respectively $(p<0.05$ and $p<0.01)$ in patients showing clinical deterioration.

The individual values of EPs at the first and final investigation are plotted in figs 2 and 3 . The latencies of VEPs and SEPs were pooled together if there was no significant change from the first to the final examination independent of the clinical findings. The average increase in latency of the VEPs that occurred in nine patients in whom the disease progressed was $22 \pm 2.3 \mathrm{~ms}(\mathrm{n}=22)$, of the SEPs following median nerve stimulation which occurred in four patients was $11.9 \pm 3.8 \mathrm{~ms}(\mathrm{n}=7)$. Moreover, in seven patients there was an average increase in latency of $25 \pm 5.1 \mathrm{~ms}(\mathrm{n}=11)$ following tibial nerve stimulation.

In three patients with clinical progression the latency of evoked potentials following pattern reversal stimulation was shorter at the final than at the first examination (fig 2), in one following both median and tibial nerve stimulation and in two 


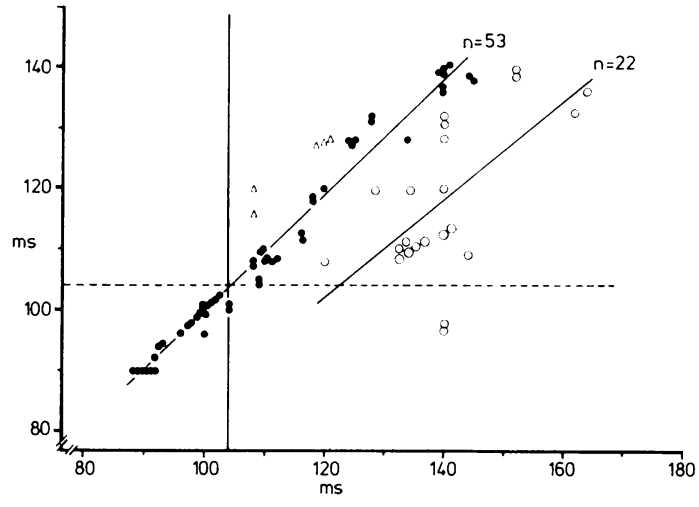

Fig 2 Latency in ms of P100 to pattern reversal stimulation in patients with clinical progression and increased conduction abnormalities (open circles). Abscissa and ordinate latency at the final and first investigation respectively. The dots indicate latencies in patients without progression either clinically or electrophysiologically. The triangles represent values from three patients in whom there was a shortening of the latency of P100 from the first to the last investigation in spite of clinical progression. The stippled lines indicate the upper $99 \%$ confidence limits of P100 latency in normal subjects. The thick lines are the regression lines for values in patients who progressed clinically and electrophysiologically $(n=22)$ and for patients without progression either clinically or electrophysiologically $(n=53)$.

others after stimulation of either the median or the tibial nerve (fig 3 ). The mean decrease in latency was $9 \mathrm{~ms}, 4.3 \mathrm{~ms}$ and $9.5 \mathrm{~ms}$ for the VEP, SEP $M$ and SEP $T$ respectively. In most patients without any symptoms and signs of progression clinically there was no change in latency of EPs between the first and final investigation. However, in one patient the latency of the SEP increased 13 and $8 \mathrm{~ms}$ respec- tively following tibial nerve stimulation on the left and right side, and in another the latency decreased 5 and 6 ms respectively (fig 3 ).

There was electrophysiological evidence of new lesions within the CNS in four patients of whom two were originally classified as probable and two as

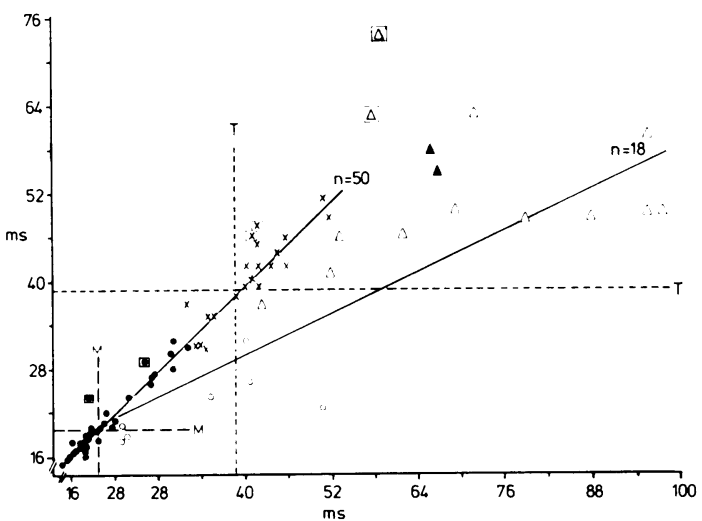

Fig 3 Latency in ms to onset of cortical evoked potentials following median and tibial nerve stimulation at the first (ordinate) and final investigation (abscissa). Dots and open circles denote latency of SEPs evoked by stimulation of the median nerve at wrist, crosses and triangles those following tibial nerve stimulation at ankle. The open circles and triangles are from patients with clinical progression; the other symbols represent patients without progression either clinically or electrophysiologically. $\mathrm{\square} \otimes$ denote shortening of latency from first to final investigation. denotes increase of latency of SEP after tibial nerve stimulation in one patient without clinical progression. The stippled lines indicate the $99 \%$ upper confidence limits for latencies of SEPs evoked by median $(M)$ and tibial $(T)$ nerve stimulation in normal subjects. The thick lines are the regression lines for EPs $(n=18)$ in patients with clinical progression and in those without progression either clinically or electrophysiologically $(n=50)$.

Table 4 Mean latency \pm SE of P100 evoked by pattern reversal stimulation and onset of SEP evoked by stimulation of the median $(M)$ and tibial $(T)$ nerves in 21 patients with suspected multiple sclerosis

\begin{tabular}{|c|c|c|c|c|c|c|c|}
\hline & Clinically & & & & & & \\
\hline & & Progressed & No. & & Unchanged & $N_{O}$ & \\
\hline \multirow{2}{*}{ VEP (P100) } & first exam. & $116 \pm 3 \cdot 1$ & \multirow{2}{*}{$24^{*}$} & \multirow{2}{*}{ (12) } & $108 \pm 4 \cdot 1$ & \multirow{2}{*}{16} & \multirow{2}{*}{ (8) } \\
\hline & final exam. & $127 \pm 3.9$ & & & $109 \pm 3.0$ & & \\
\hline \multirow{2}{*}{ SEP M (onset) } & first exam. & $21 \cdot 2 \pm 0 \cdot 9$ & \multirow{2}{*}{26} & \multirow{2}{*}{ (13) } & $21 \cdot 6 \pm 1 \cdot 6$ & \multirow{2}{*}{$14 \dagger$} & \multirow{2}{*}{ (7) } \\
\hline & final exam. & $23 \cdot 9 \pm 1 \cdot 8$ & & & $21 \cdot 5 \pm 1 \cdot 5$ & & \\
\hline \multirow{2}{*}{ SEP T (onset) } & first exam. & $46 \pm 2 \cdot 0$ & \multirow{2}{*}{$23 \ddagger$} & \multirow{2}{*}{ (12) } & $44 \pm 2 \cdot 8$ & \multirow{2}{*}{$13 \S$} & \multirow{2}{*}{ (7) } \\
\hline & final exam. & $58 \pm 4.4$ & & & $45 \pm 3 \cdot 2$ & & \\
\hline
\end{tabular}

Figure in brackets $=$ number of patients,${ }^{*}$ VEP absent unilaterally in two patients, $\uparrow$ SEP M not performed in one patient, $¥$ SEP T absent bilaterally and unilaterally in each one patient, §SEP T absent unilaterally in one patient and not performed in one. For each patient there is two values for VEPs and SEPs following median and tibial nerve stimulation. 
Table 5 Mean latency $\pm S E$ of evoked potentials in 13 suspected multiple sclerosis cases with and eight without clinical signs and symptoms of progression

\begin{tabular}{|c|c|c|c|c|c|c|c|c|c|c|}
\hline \multirow{4}{*}{$\begin{array}{l}E P \\
\operatorname{VEP}(\mathbf{P} 100)\end{array}$} & \multirow{4}{*}{$\begin{array}{l}\text { Latency } \\
\begin{array}{l}\text { increased } \\
\text { unchanged or } \\
\text { decreased }\end{array}\end{array}$} & \multicolumn{9}{|c|}{ Clinically } \\
\hline & & \multicolumn{5}{|c|}{ Progressive (13 patients) } & \multicolumn{4}{|c|}{ Non-progressive (8 patients) } \\
\hline & & \multicolumn{2}{|c|}{ first exam. } & \multicolumn{2}{|c|}{ final exam. } & \multirow{2}{*}{$\begin{array}{l}\text { No. } \\
12^{*}(9) \\
12 \dagger\end{array}$} & \multirow{2}{*}{$\begin{array}{l}\text { furst exam. } \\
\overline{109} \pm 2.7\end{array}$} & final exam. & \multicolumn{2}{|l|}{ No. } \\
\hline & & $\begin{array}{l}118 \\
115\end{array}$ & $\begin{array}{l} \pm 3.9 \\
\pm 5.0\end{array}$ & $\begin{array}{l}141 \\
114\end{array}$ & $\begin{array}{l} \pm 2.8 \\
\pm 4.9\end{array}$ & & & $\overline{109}$ & 16 & (8) \\
\hline SEP M (onset) & $\begin{array}{l}\text { increased } \\
\text { unchanged or } \\
\text { decreased }\end{array}$ & $\begin{array}{l}23 . \\
20 .\end{array}$ & $\begin{array}{l} \pm 1.9 \\
\pm 1.0\end{array}$ & $\begin{array}{l}33 . \\
20\end{array}$ & $\begin{array}{l} \pm 4 \cdot 2 \\
\pm 1 \cdot 1\end{array}$ & $\begin{array}{rr}7 & (4) \\
19 & (10)\end{array}$ & $\overline{2} 1.6 \pm 1.6$ & $\overline{2} 1.5 \pm 1 \cdot 5$ & $14 \ddagger$ & (7) \\
\hline SEP T(onset) & $\begin{array}{l}\text { increased } \\
\text { unchanged or } \\
\text { decreased }\end{array}$ & $\begin{array}{l}49 \\
44\end{array}$ & $\begin{array}{l} \pm 2 \cdot 2 \\
\pm 3 \cdot 1\end{array}$ & $\begin{array}{l}73 \\
43\end{array}$ & $\begin{array}{l} \pm 5.9 \\
\pm 2.3\end{array}$ & (7) & $\pm 2 \cdot 8$ & $\pm 2 \cdot 1$ & $\begin{array}{c}2 \\
11 \S\end{array}$ & (1) \\
\hline
\end{tabular}

Figure in brackets $=$ number of patients. They do not necessarily add up to the total for the subgroups of EPs as a patient can be represented in both.

* One value for each eye. No response from one eye. †One patient had one eye removed. $\ddagger$ Not performed in one patient, §No response to simulation of the left tibial nerve in one patient, not performed in another patient on either side.

possible multiple sclerosis cases, all four deteriorated clinically. In one of the probable cases who had delayed EPs bilaterally following visual and tibial nerve stimulation at both the first and the final investigation, the cortical SEP following left and right median nerve stimulation were delayed at the reexamination. In the other probable case who had delayed responses to visual and median nerve stimulation unilaterally and to tibial nerve stimulation bilaterally at the first investigation the VEP from the unaffected eye was in addition delayed at the follow-up. In one possible multiple sclerosis case who only had abnormal VEPs bilaterally at the first examination, the EP following left tibial nerve was in addition delayed at the last investigation. Finally, in the other possible case in whom all EPs except that following left tibial nerve stimulation were abnormal when the patient was first seen, all six EPs were abnormal at the follow-up.

\section{Discussion}

The most important consequence of evoked potential techniques in the diagnosis of multiple sclerosis is their ability to reveal clinically silent lesions within the CNS. An increased content of immunoglobulin $G$ in the cerebrospinal fluid also supports the diagnosis. ${ }^{5-7}$ Combining the results of both tests may therefore increase the diagnostic yield. We have suggested a reclassification of suspected multiple sclerosis cases with abnormal EPs when the intracerebral synthesis of $\mathrm{IgG}$ expressed by the ratio of CSF serum IgG and CSF serum albumin was also increased. ${ }^{\prime}$

In the present follow-up study of 21 patients with suspected multiple sclerosis we found that 13 of 16 patients all of whom fulfilled the proposed criteria for a reclassification of their disease progressed clin- ically. Moreover, in 10 of these patients there was also evidence of progressive conduction abnormalities within the CNS, and in four there was evidence of new lesions. Of the 13 patients with clinical progression four belonged to the group of probable cases and nine to the possible cases. Of the seven patients classified as probable cases three had a normal IgG index and none of these three patients progressed clinically. All the five possible cases without clinical progression had a mild ${ }^{1}$ increased IgG index and two of them had normal EPs (fig 1).

Deterioration occurred more often among patients with a moderate to large increase of intracerebral IgG synthesis than among those with a normal or slightly increased IgG index. This is in agreement with findings of Olssson et al, ${ }^{8}$ who stated that CSF immunoglobulin abnormalities occurred more often and were more pronounced in patients with a more malignant course of the disease. However, our conclusion must be tentative as the number of patients is small and we have no followup of suspected multiple sclerosis cases in whom both EP and IgG index are normal. The progression of the disease was not related to age of the patient, age at onset or time elapsed since the first attack.

Although an abnormal EP in multiple sclerosis patients usually remains delayed, normalisation of EP latency with clinical remission has been reported..$^{9-13}$ We did not observe any such change in our material. On the other hand in 11 of 21 patients there was an increase of the EP latency from the first to the final investigation. All but one of these 11 patients belonged to the group in whom the disease progressed. On the contrary in seven patients we found a shorter EP latency at the final investigation than at the first. Six of these patients had a progressive course and one did not. Thus changes in latency of EPs between the two investigations occurred in 
18 patients; in three of these the latency of the VEP increased, whereas the latency of the SEP following either median or tibial nerve stimulation decreased. On account of changes in EP latencies it was not possible to predict the course of the disease.

To our knowledge there is no follow-up study of suspected multiple sclerosis cases similar to the present investigation. Matthews et al ${ }^{14}$ examined 39 patients with a single acute episode resembling multiple sclerosis and found delayed VEP in only two, both of whom at follow-up entered into one of the diagnostic categories of multiple sclerosis. The fate of the other 37 patients remains unknown. In another series of 96 possible cases of whom 27 patients $(28 \%)$ had an abnormal VEP, subsequent clinical development and the result of other investigative procedures were compatible with the original diagnostic classification. ${ }^{d s}$ However, there was no information as to the follow-up period, and the authors stated that a longer observation time was necessary to evaluate the diagnostic significance of the findings. In this connection it is important to emphasise that in none of our three non-progressive multiple sclerosis cases who fulfilled our diagnostic criteria for a reclassification had the follow-up examination (including laboratory test to exclude metabolic disorders and CT scan or myelography when indicated) revealed any other structural pathology as a cause of their symptoms and signs.

The present results seem to justify a reclassification of suspected multiple sclerosis cases into a higher diagnostic class at the first consultation when EP techniques provide evidence of more lesions than clinical examination, and if the $\mathrm{IgG}$ index also is increased.

This investigation was supported by the Danish Medical Research Council. The technical assistance of Kamma Astow, Erna Petersen, Jytte Schmidt, and Birthe Winther is gratefully acknowledged.

\section{References}

1 Trojaborg W, Böttcher J, Saxtrup O. Evoked potentials and immunoglobulin abnormalities in multiple sclerosis. Neurology (NY) 1981;31:866-71.
${ }^{2}$ Halliday AM, McDonald WI. Diagnosis and classification of multiple sclerosis. $\mathrm{Br}$ Med Bull 1977;33:4-8.

${ }^{3}$ Trojaborg W, Petersen, E. Visual and somatosensory evoked cortical potentials in multiple sclerosis. $J$ Neurol Neurosurg Psychiatry 1979;42:323-30.

4 Petersen L, Trojaborg W. Visual, auditory and somatosensory pathway involvement in hereditary cerebellar ataxia, Friedreich's ataxia and familial spastic paraplegia. Electroencephalogr Clin Neurophysiol 1981;52:283-97.

${ }^{5}$ Link H, Møller E, Norrby E, et al. Immunoglobulin abnormalities in spinal fluid in multiple sclerosis. Acta Neurol Scand 1977;Suppl 63:173-89.

- Link H, Tibling G. Principles of albumin and IgG analysis in neurological disorders: III Evaluation of IgG synthesis within the central nervous system in multiple sclerosis. Scand J Clin Lab Invest 1977;37:397-401.

7 Christensen O, Clausen J, Fog T. Relationships between abnormal IgG index, oligoclonal bands, acute phase reactants and some clinical data in multiple sclerosis. $J$ Neurol 1978;218:237-44.

${ }^{8}$ Olsson J-E, Link H, Müller R. Immunoglobulin abnormalities in multiple sclerosis; relation to clinical parameters: disability, duration and age of onset. $J$ Neurol Sci 1976;27:233-45.

${ }^{9}$ Asselman P, Chadwick DW, Marsden CD. Visual evoked responses in the diagnosis and management of patients suspected of multiple sclerosis. Brain 1975;98:261-82.

${ }^{10}$ Namerow NS. Somatosensory evoked responses in multiple sclerosis patients with varying sensory loss. Neurology (Minneap) 1968;18:1197-1204.

11 Kimura J. Electrically elicited blink reflex in diagnosis of multiple sclerosis. Review of 260 patients over a seven-year period. Brain 1975;98:413-26.

12 Halliday AM, McDonald WI. Pathophysiology of demyelinating disease. Br Med Bull 1977;33:21-7.

${ }^{13} \mathrm{Kjær}$ M. Variations of brain stem auditory evoked potentials correlated to duration and severity of multiple sclerosis. Acta Neurol Scand 1980;61:157-66.

${ }^{14}$ Matthews WB, Small DG, Small M, Pountney E. Pattern reversal evoked visual potential in the diagnosis of multiple sclerosis. J Neurol Neurosurg Psychiatry 1977;40:1009-14.

is Aminoff MJ, Ochs AL. Pattern-onset visual evoked potentials in suspected multiple sclerosis. $J$ Neurol Neurosurg Psychiatry 1981;44:608-14. 